\title{
On the dependence of magnetic line ratios on time and spatial scales
}

\author{
Mikhail L. Demidov \\ Institute of Solar-Terrestrial Physics, P.O. Box 291, 664033 Irkutsk, Russia \\ email: demid@iszf.irk.ru
}

\begin{abstract}
Comparison of magnetic fields measurements made in different spectral lines and observatories is an important tool for diagnostics of magnetohydrodynamic conditions in the solar atmosphere. But there is a deficit of information about the dependence of results on detailed position on the solar disk, spatial resolution and time. In this study these issues are discussed in application to the solar large-scale and Sun-as-a-star magnetic fields observations.
\end{abstract}

Keywords. Sun: magnetic fields - sunspots - Sun: activity

Comparisons of solar magnetic field observations in different spectral lines (e.g. Howard \& Stenflo, 1972; Stenflo, 1973; Ulrich, 1992) and in different observatories (Wenzler et al., 2004; Tran et al., 2005; Demidov et al., 2008) are of crucial importance for understanding of the solar magnetism nature. According to different studies there are some differences in the values of magnetic strength ratios (MSR) even when the same combinations of spectral lines are used. There are some results concerning the dependence of MSR for several cases on filling factor, amount of flux, or center-to-limb angle. Complicated and sometimes rather unexpected character of spatial distribution of MSR across the solar disk when different combinations of spectral lines and observatories are used, is shown in the recent paper (Demidov et al., 2008). The main objective of this study is to explore the possible dependence of MSR on time and spatial resolution in application to the large-scale (LSMF) and solar mean (SMMF) magnetic fields.

Comparison of SMMF measurements, made in different combinations of four spectral lines in the vicinity of Fe I $\lambda 525.02 \mathrm{~nm}$ is shown in Fig. 1 (left panel). To obtain this result, the Stokes-meter simultaneous observations made at the STOP telescope at the Sayan Solar Observatory (SSO) during 1999-2006 years were used. The sunspot numbers (SN) data (http://www.ngdc.noaa.gov) are shown in the right panel of figure 1. For all combinations of spectral lines there are only very small variations of MSR with time. When the SN numbers decreased in more then six times from 2000 to 2006, there is only a small decreasing of MSR. Figure 2 shows the variations during the same time interval of the mean (absolute values) SMMF strength in the case of the J.Wilcox Solar Observatory (WSO) measurements (left panel) and of the $B_{W S O} / B_{S S O}$ SMMF strength ratio (right panel). Naturally, SSO data are in the Fe I $\lambda 525.02 \mathrm{~nm}$ spectral line.

There are some indications that MSR could depend on spatial resolution used in observations (Ulrich, 1992; Demidov et al., 2008). According to Ulrich (1992), the reduction factor (by which the MWO magnetic fields observed in the Fe I $\lambda 525.02 \mathrm{~nm}$ spectral line should be multiplied in comparison to observations in the Fe I $\lambda 523.29 \mathrm{~nm}$ ) is increasing with decreasing of spatial resolution: $R=4.5$ for the disk center for the resolution of 20 arc seconds, and $R=3.5$ for the case of 5 arc seconds. But this result contradicts to the Howard and Stenflo (1972) and recent author's results. According to Howard and 

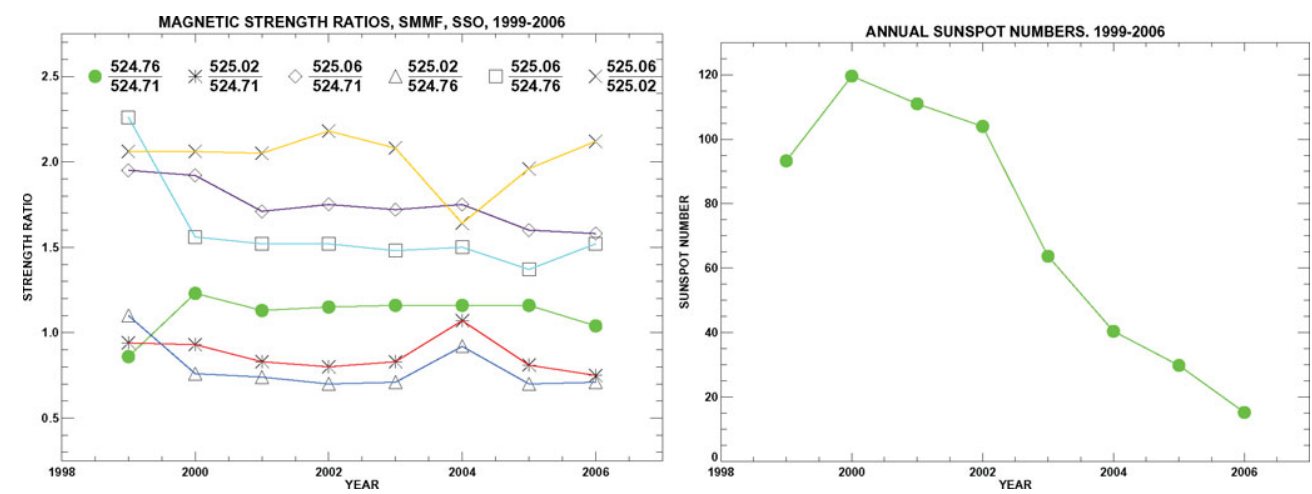

Figure 1. Left panel: strength ratios for the SMMF Stokes-meter observations (Sayan Solar Observatory STOP telescope) in four spectral lines near Fe I $\lambda 525.02 \mathrm{~nm}$. Right panel: the sunspot numbers for 1999-2006 years.
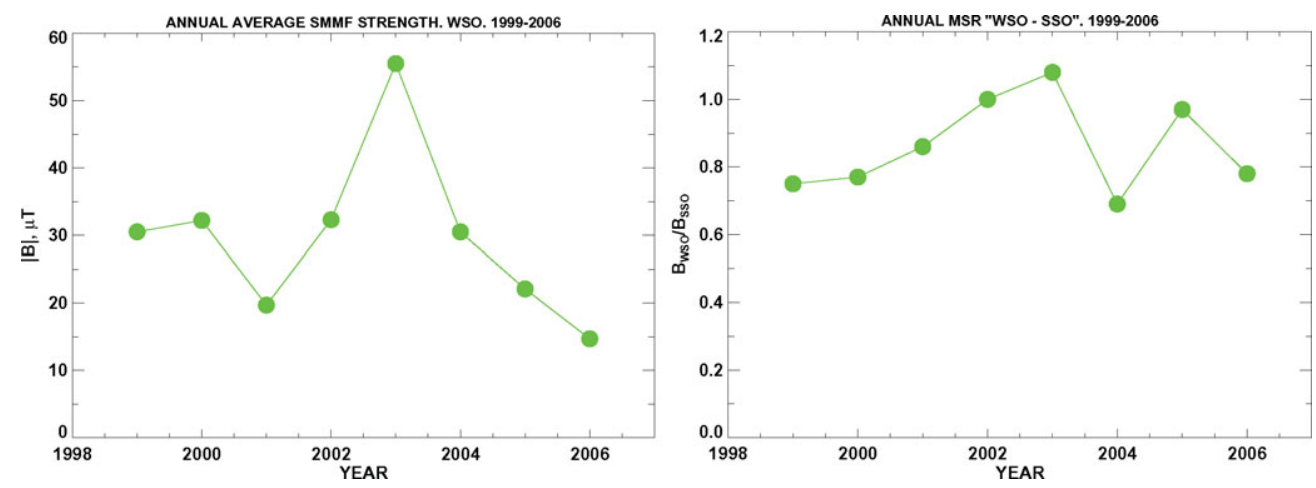

Figure 2. Variation with time of the mean absolute SMMF strength according to WSO data (left panel), and of the WSO/SSO SMMF strength ratio (right panel).

Stenflo (1972) $R=1.8$ for the disk center, and $R=3.0$ (spatial resolution about 100 arc seconds) according to the STOP SSO observations.

Numerous LSMF and SMMF observations on the STOP telescope give the following MSR numbers for the Fe I $\lambda 524.70 \mathrm{~nm}$ - Fe I $\lambda 525.02 \mathrm{~nm}$ combination: $R=0.83$ for the LSMF, and $R=0.93$ for the SMMF. In the paper (Demidov et al., 2008) the possibility is suggested that the dependence of MSR on spatial resolution is the consequence of non-linearity of regression coefficients on magnetic field strength (filling factor).

\section{Acknowledgements}

The author is grateful to the IAUS 259 SOC and to the RFBR for partial financial support.

\section{References}

Demidov, M. L., Golubeva, E. M., Balthasar, H., Staude, J., \& Grigoryev, V. M. 2008, SP 250, 279

Howard, R. \& Stenflo, J. O. 1972, SP 22, 402

Stenflo, J. O. 1973, SP 32, 41

Tran T., Bertelle L., Ulrich, R. K., \& Evans, S. 2005, ApJS 156, 295

Ulrich, R. K. 1992, ASP Conf. Ser. 26, 265

Wenzler, T., Solanki, S. K., Krivova, N. A., \& Fluri, D. M. 2004, A\&4A 427, 1031 